MATEC Web of Conferences 22,02001 (2015)

DOI: $10.1051 /$ matecconf/ 20152202001

(c) Owned by the authors, published by EDP Sciences, 2015

\title{
Research on the Substation Intelligent Protection System for Operations Personnel Based on the UWB Positioning Technology
}

\author{
Zhen $\mathrm{Yu}^{*}$ \\ State Grid Smart Grid Research Institute, Beijing, China \\ Zhiyao He \\ North China Electric Power University,Baoding, Hebei, China
}

\begin{abstract}
In order to improve the safety of the operations personnel, the authors designed the substation intelligent operations protection system, studied the positioning technology, three-dimensional geographic information technology and other key technologies, proposed the technical solutions of the positioning base station, the positioning label and the power supply system, and then derived the technical architecture of the substation intelligent operations protection system, which consists of the infrastructure layer, the support layer, the business layer and the presentation layer. These four layers are used to achieve the task management, real-time monitoring warning sign, area delineation, track playback and other functions.
\end{abstract}

Keywords: power safety; UWB positioning technology; three-dimensional geographic information systems; operations personnel; illegal operations

\section{INTRODUCTION}

Currently, the electricity security situation remains stable, but the personal security situation remains bad [1-3]. During the span of 11 th five-year plan, 88 personal casualty accidents of the country's electricity production have killed 112 people. Such accidents exposed that the part of the safety production unit had weak link and loopholes in management, such as the shallowness of the staff safety consciousness, undemanding rules and regulations, illegal command and operation; security management which not reaches the designated position with undemanding and unspecific organization; incorrect use of tools and instruments. And the auxiliary equipment management does not reach the designated position with improper fault emergency treatment measures [4-5]. According to a lot of statistics and analysis, $60 \%-90 \%$ of the electricity accidents are mainly caused by people's unsafe factors.

By studying the positioning technology, three-dimensional geographic information system and other information technologies, we can achieve the automatic monitoring to the safety behavior of operation field personnel and do alarm before the operations personnel violation. It has theoretic importance and outstanding application value to guard against illegal acts, prevent or reduce the personal injury accidents and other dangerous accidents of the power system.

\section{SYSTEM PRINCIPLES}

System mainly adopts the positioning technology and the intelligent video analysis technology to realize

*Corresponding author: yuzhenbj@,163.com data collection, analysis, and timely send illegal operations information to illegal workers and supervisor. The principle is shown in Figure 1.

\subsection{Data acquisition}

Data acquisition mainly includes the location information acquisition and the image data acquisition. The location information is collected through the positioning label worn by operations personnel. Then the positioning label will be used to communicate with four positioning base station around substation, and generate the position coordinates of operations personnel in the data acquisition server, expressed as $(\mathrm{X}, \mathrm{Y}, \mathrm{Z})$ in geocentric rectangular coordinate system after TDOA calculation.

The image information acquisition mainly takes advantage of existing substation video monitoring system. The video analysis server preprocesses video data (4cif resolution) from the video monitoring terminal, and then detects moving targets by the background reduction method [6] and the optical flow reduction method [7-8] to generate the target objects in data acquisition server. The system stream is shown in Figure 2.

\subsection{Alarm information generation}

Any equipment within the substation has a fixed three-dimensional coordinate after the three-dimensional panorama simulation modelling of substation scene. When workers get close to the charged equipment at a distance which is less than the safe distance, the system will produce position alarm information. Location information is generated by alarm conditions: 


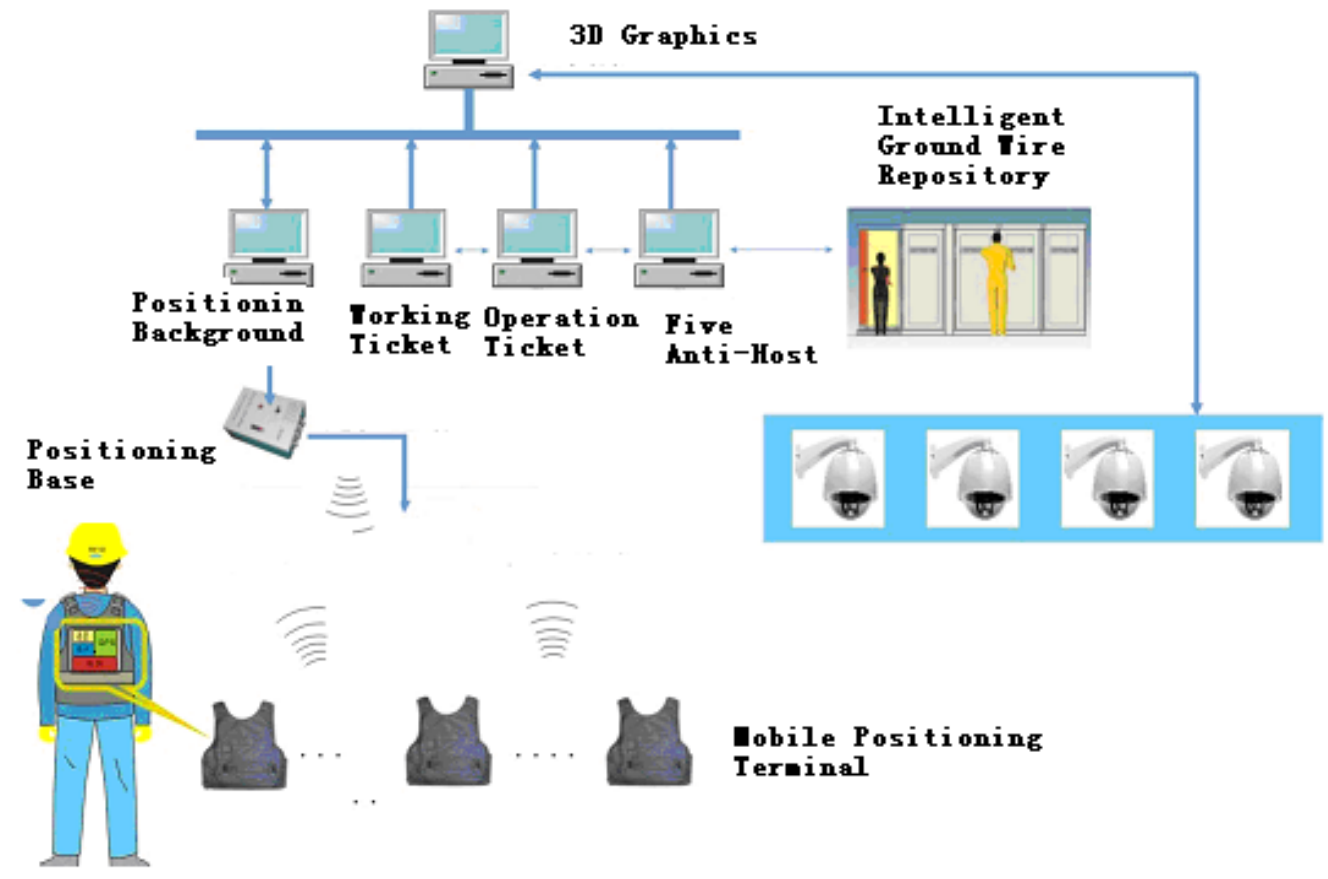

Figure 1. Diagram of the system principle

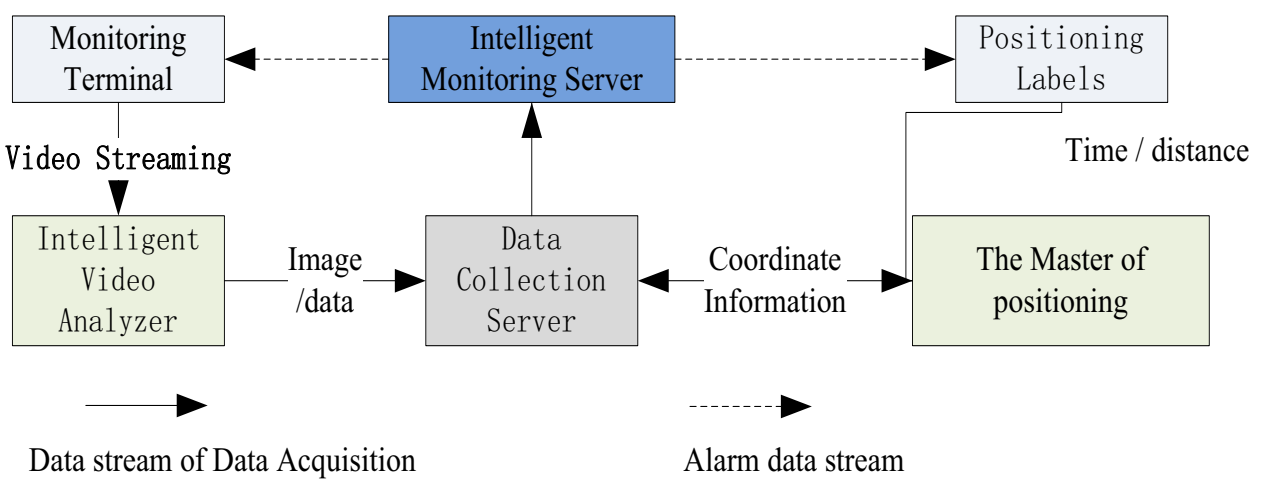

Figure 2. Flow of data acquisition and processing

$\sqrt[2]{\left(\mathrm{X}-\mathrm{X}_{0}\right)^{2}+\left(\mathrm{Y}-\mathrm{Y}_{0}\right)^{2}+\left(\mathrm{Z}-\mathrm{Z}_{0}\right)^{2}}<a$

Where $(\mathrm{X} 0, \mathrm{Y} 0, \mathrm{Z} 0)$ is the electric equipment location coordinate; "a" is the safe distance of equipment. Location alarm includes near charged equipment, wrong operation interval, wrong operation and other violation alarm.

Another kind of alarm information is the video alarm information. The video data flows through the data collector can generate target object which is then compared to the source target from violation rule base. If the two are matched, the target object is considered to be illegal. For example, when workers are not wearing safety helmet, the target object of collection server is non-wearing safety operations personnel, and there is non-wearing safety helmet personnel (source target) in violation rule base, so the two are matched. The situation for video alarm includes personnel without wearing a helmet, no responsible operation personnel in the work area, personnel outside the safety area or cross the picket line and so on.

\subsection{Alarm notification}

The intelligent protection server sends the alarm message in the form of "sound" and "image" to the illegal personnel and operations monitor in the operations site, 


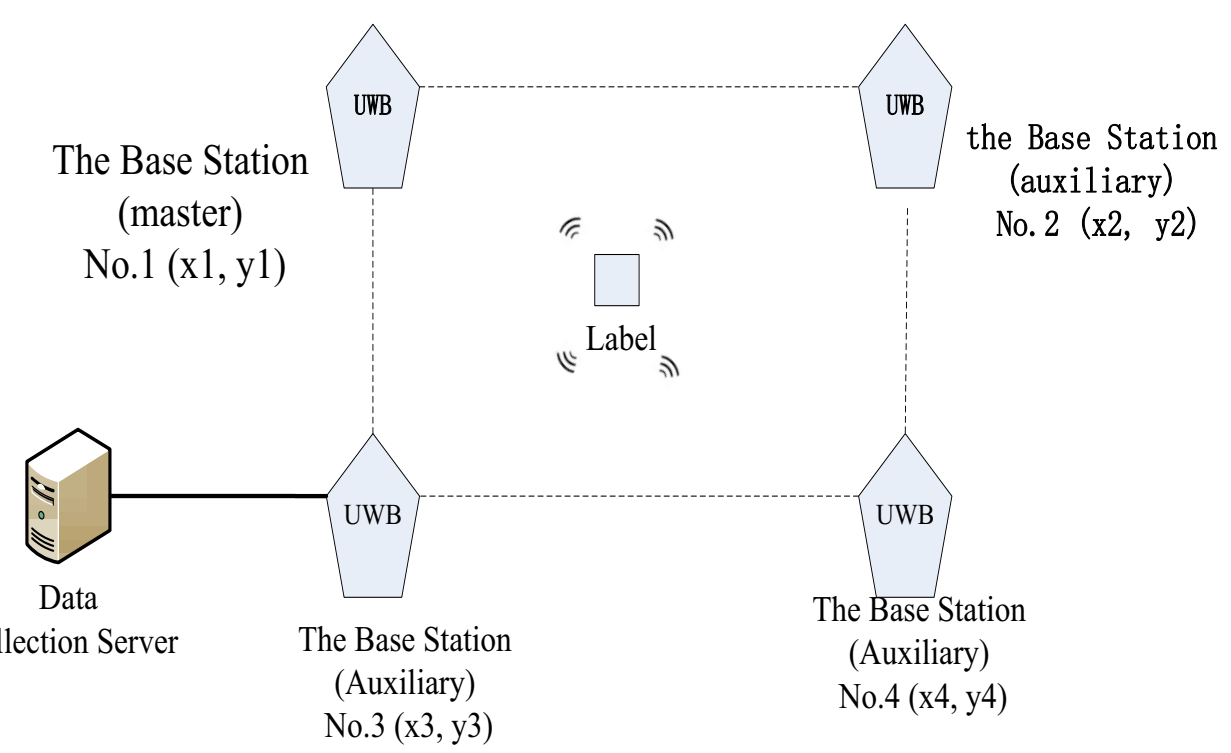

Figure 3. Sketch map of UWB Positioning system

\section{hntenna}

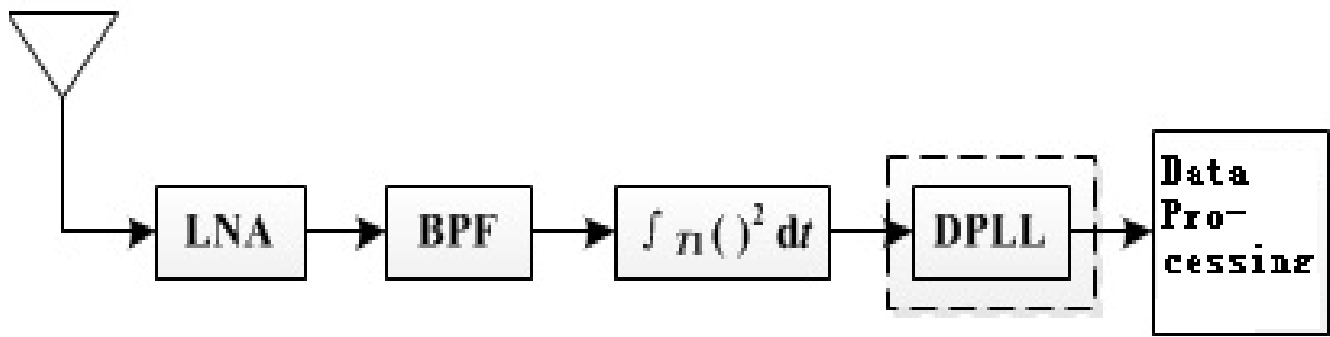

Figure 4. Block diagram of the base station location

so does the substation duty personnel at the same time, and system will also send the illegal information to the database for the future illegal analysis.

\section{KEY TECHNOLOGIES}

\subsection{UWB positioning technology}

The system adopts UWB (Ultra Wide Band) wireless positioning technology. UWB is a high-speed, low-cost and low-power-consumption new wireless communication technology [9-11]. The UWB signal is a pulse signal of which the bandwidth is more than $500 \mathrm{MHz}$ or the ratio of fundamental wave bandwidth and carrier frequency is greater than 0.2 , which has a wide band range. The UWB frequency band of FCC regulations is from $3.1 \mathrm{GHz}-10.6 \mathrm{GHz}$, and limit the transmit power of the signal below $41 \mathrm{dBm}$ [9]. The UWB positioning technology can meet the require- ment of precise positioning requirement in substation range, which the positioning accuracy can reach $30 \mathrm{~cm}$, along with low-cost, anti-multipath interference ability, and strong penetration advantages. The UWB positioning system is composed of positioning sensors, positioning labels and other components. Each positioning unit consists of more than four positioning sensors as shown in Figure 3.

3.2 Three-dimensional geographic information system $(3 D$ GIS

3D GIS which is capable of true three-dimensional description, visualization, analysis and management refers to space object of a specific region, which should provide a high-dimensional visual effect, satisfy the geographic information system of a wide range of city architecture, and provide comprehensive spatial information query and analysis function which are similar to two-dimensional GIS. Due to the high- 


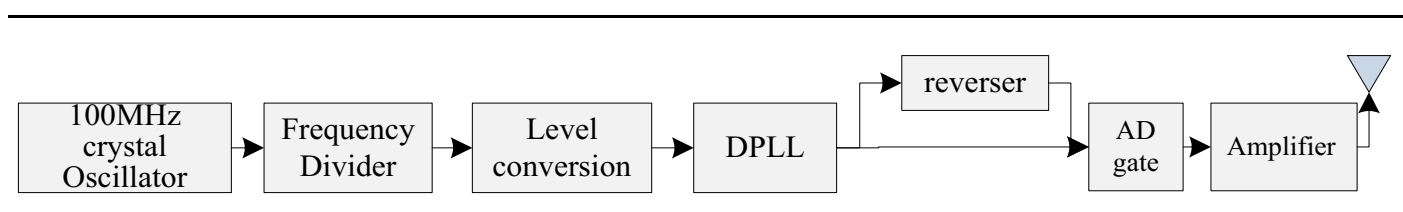

Figure 5. Diagram of a transmitter tag

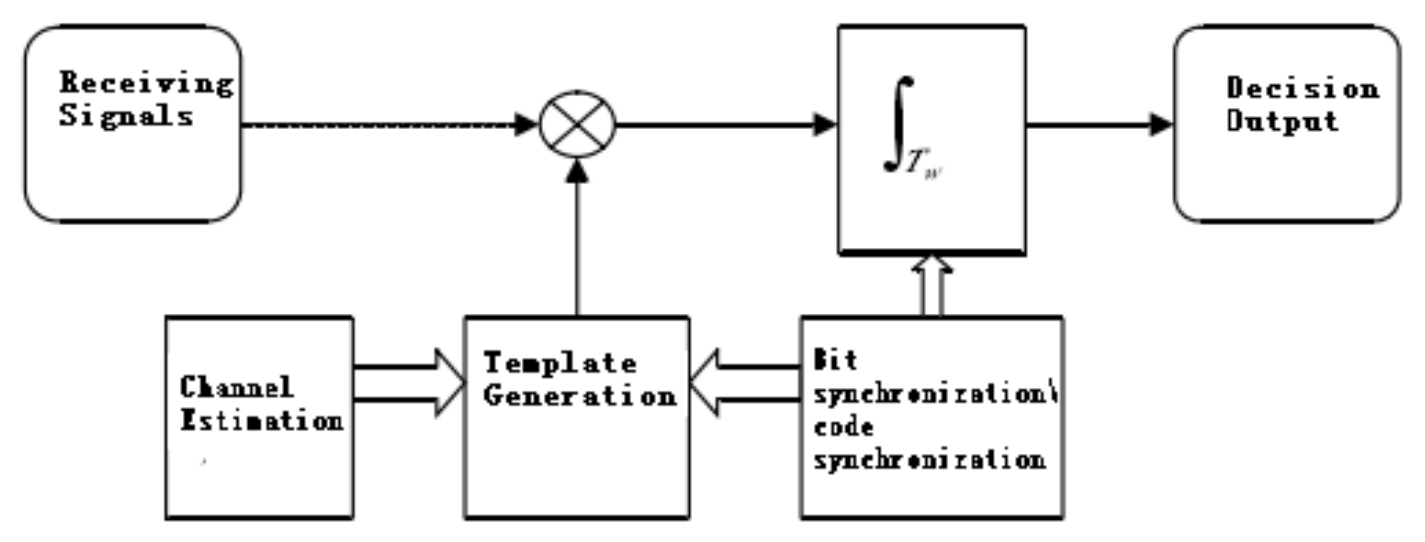

Figure 6. Block diagram of the alarm signal receiver module light attention to the personnel space and the $\mathrm{Z}$-axis of space three-dimensional coordinates for the safety of electric power production personnel management and the objective of the study, this project must adopt three-dimensional GIS rather than with two-dimensional GIS only. On the basis of analysing the characteristics of the electric power production on-site, it is concluded that the use of the substation three-dimensional simulation graphics platform satisfied the safety management of $\mathrm{Z}$-axis coordinate and a high demand of data for personnel activities.

\section{POSITIONING EQUIPMENT}

\subsection{Design of the positioning base station}

The base station receiver consists of antenna, low noise amplifier (LNA), band pass filter (BPF), square integrator, and digital phase-locked loop (DPLL). Being different from the traditional energy detection receiver, this receiver system introduced the design of digital phase-locked loop. Because the phase-locked loop is equivalent to a narrow-band filter, which has strong antinoise performance, it can effectively improve the robustness of the receiver system to some extent without increasing the signal transmission power condition, and effectively reduce the probability of missed detection and false alarm.

\subsection{Positioning labels}

4.2.1 Design of the positioning labels pulse transmitter

The pulse transmitter of positioning label consists of four parts: the trigger signal generating circuit, the level compatible circuit, the competition adventure pulse generating circuit, and the amplifier circuit.

\subsubsection{Hazard alarm signal receiver module}

Reception process: Through a channel estimation, the bit and code synchronization algorithm is used to generate the local template; multiplying the template with the received signal; and then sending into judgment after time integration; at last, a judgment output happens in the end.

UWB system adopts nanosecond narrow pulses as transmission signals. The principles of signal reception module of the position label are to use a set of correlators, and each multipath component uses one correlation receiver, which makes correlation calculation with one of the received multipath components signals. Then these correlation outputs of the receiver are weighted according to their relative strength, and the weighted outputs of various quarters are combined into a output signal.

\subsection{Power supply technology of the UWB positioning unit}

There are two methods for the power supply of position sensors: The first one is to use the power adapter, and the second is to use the POE switch power supply.

Power adapter is divided into two modes: One is the centralized power supply, which means the power supply is from the same place; and another one is the distributed power supply, that is, all positioning sensors obtain power from the vicinity of the installation location. From the perspective of interference effects, 
ICETA 2015

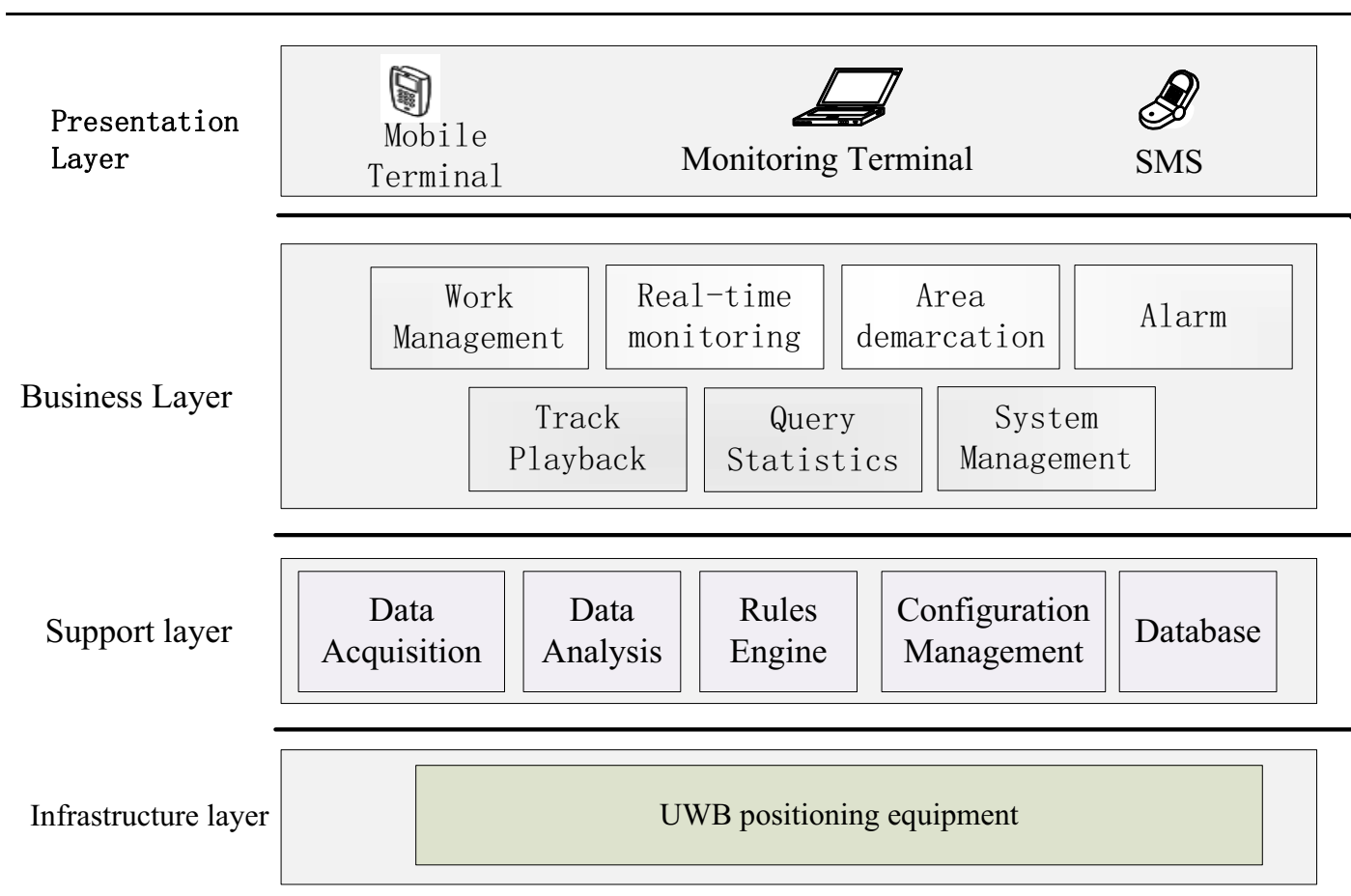

Figure 7. System technical architecture

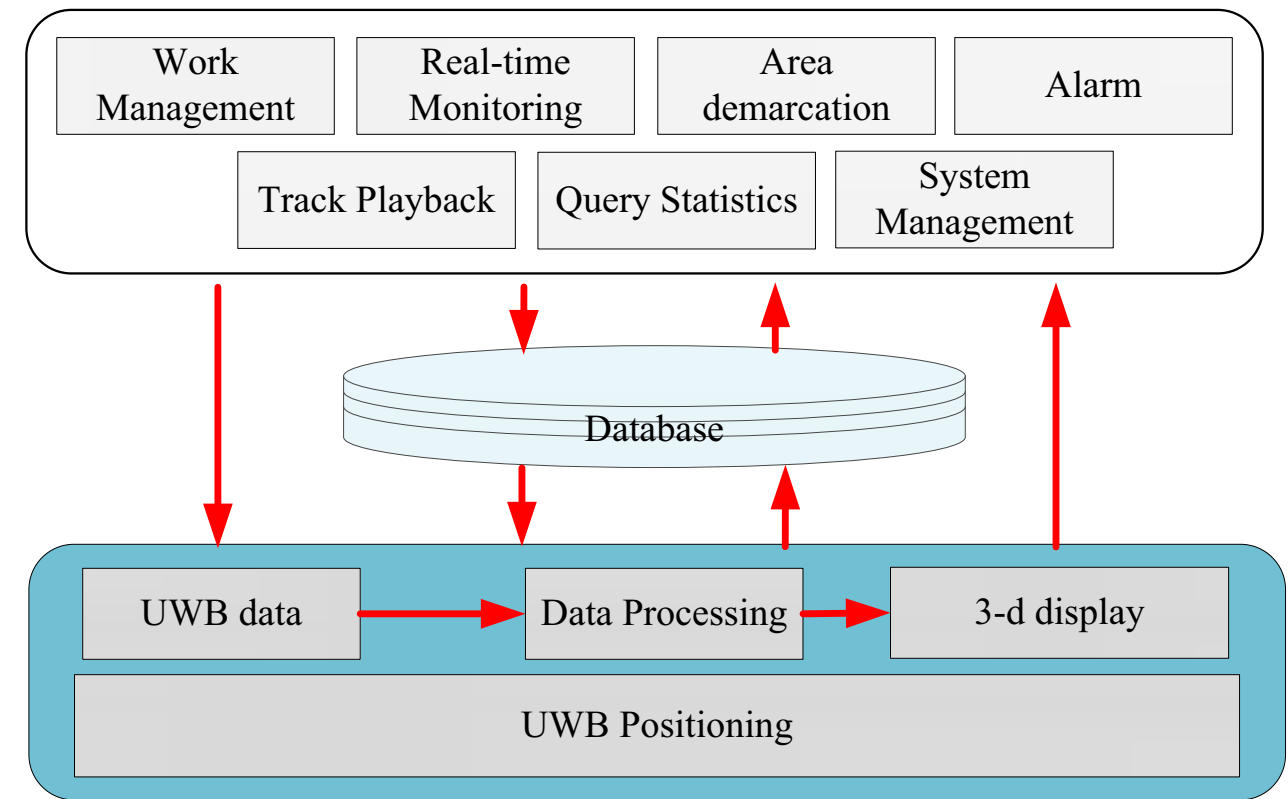

Figure 8. System function architecture

the centralized power can basically eliminate the reference potential differ impact throughout. The overall outage risk of distributed power supply risk is relatively scattered, but there exists a constant management, which is high-cost and not easy to maintain.

\section{DESIGN OF THE INTELLIGENT PROTECTION SYSTEM}

\subsection{Technical architecture}

To ensure the scalability, reusability and maintaina- 
Table 1. Comparison of power supply

\begin{tabular}{|l|l|l|l|l|}
\hline Power supply & Costs & Maintain & Power outage risk & Management \\
\hline Power adapter & High & Inconvenient & Dispersion & Inconvenient \\
\hline POE power supply & low & Convenient & Centralized & Convenient \\
\hline
\end{tabular}

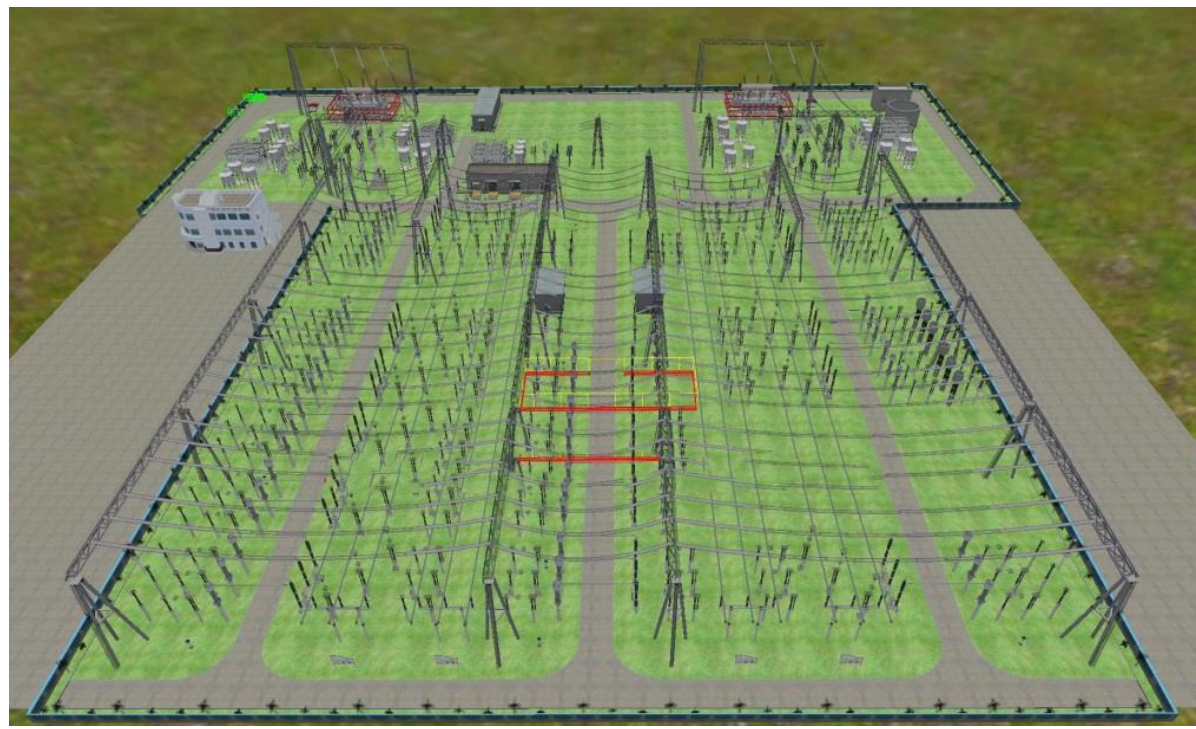

Figure 9. Interface of real-time monitoring

bility, the system adopts the concept of hierarchical design. System can be divided into infrastructure layer, support layer, business layer and presentation layer. The infrastructure layer including the front-end acquisition equipment of location information and device information, provides the underlying equipment support for the support layer; the support layer includes data acquisition, processing, storage and other underlying software platform; the business layer achieves the main function of intelligent protection system; the presentation layer which is responsible for the alarm message output and display includes the warning sound and flashing light of mobile terminals wore by workers, mobile phone alarm messages and the monitoring terminal of on duty personnel. The system architecture is shown in Figure 7.

\subsection{Functional design}

The main feature of intelligent protection system includes the task management of operations personnel, the real-time monitoring, the operations area demarcation, the danger alarm, the track playback, the statistical query and system management functions and so on. The architecture is shown in Figure 8.

\subsubsection{Task management function}

Integrated with two ticket system, the system can automatically identify the current tasks, and automatically obtain the information of work area and opera- tions personnel. The main feature of system includes the operations tasks setting and dynamic management the rapid binding and unbinding workers with label, the recording management and other functions.

\subsubsection{Real-time monitoring}

The real-time monitoring function can achieve the real-time monitoring of workers wearing the positioning label. When workers enter the work area, the system displays the worker's name and number in the three-dimensional simulation operation scenarios synchronously, and sends the basic information and the movement coordinate information to the database for future reference. Administrators can edit and exchange it by clicking on the virtual characters in the three-dimensional simulation scene.

\subsubsection{Area demarcation function}

In electric power safety operation, each work area is marked clearly in the operation ticket. Workers can only go to the designated area for operations and can't cross the work area casually. The work area demarcation function mainly includes:

1) Manual delineation: The management selects work area in the substation plan box.

2) Automatic generation: generate the working area automatically based on the work range of the work tickets.

3) The choice site generation: The supervisory per- 
sonnel selects each corner coordinates point in work area through labels in the work area, then the work area can be generated after confirmation.

\subsubsection{Danger prompt function}

When the workers step out the security zone or do other violations of the "electrical safety work order of State Grid Corporation", system can be able to alarm timely and build the last line of defence for workers. The three methods of alarming are shown as follows:

1) The voice alarm reminder of label terminal: When the violation occurs, the system will prompt alarm immediately by voice, and reminds workers immediately.

2) The voice alarm prompts of management terminal and the highlighted display of virtual monitoring area: When the violation occurs, the management terminal will prompt alarm to the substation maintenance workers through voice to stop illegal behavior timely.

3) The alarm of sending short message: The illegal work information will be sent to the mobile phone of Supervisors, operators, workers and so on.

\subsubsection{Track playback function}

The system records the operations trajectory of each worker for later analysis. System records the location of the illegal operations, and then you can view in the playback mode which includes fast, slow, and normal playback speed, and export the video.

\subsubsection{Query statistics function}

The function mainly achieve the query statistics of violations alarm, reminder times, work times, number of people and the working hours during a certain period of time, the export and print function. The function can query via the combination of conditions, including the department (team) name, the task number, the staff name, a specific date or time period.

\subsubsection{System management function}

This function can realize the management of basic data, user information, and permissions assign and system parameters, and it is the basic support module of the application system.

\section{CONCLUSION}

Combined with work site, the substation intelligent operations protection system through the study of personnel positioning, intelligent video and other technologies realizes the function of work tasks management, real-time monitoring, alarm, area delineation, and track playback. According to the tasks, the system also achieved the integration of operation and work ticket, and the intelligent management of site operations. When there are situations such as on-site forced unlock operation, workers without wearing helmets, unauthorized workers go across the security fence not from the set of entrance and exit, and wrong interval violations, the system will automatically prompt alarm, and be able to master the whole trend of operating workers in real-time.

The system which is applied in Fuzhou 500kV substation which is prompted more than 50 illegal operations in 2014, reduces the incidence of personal injury, and provides a strong support for the power safety.

In conclusion, the system provides safe and effective physical protection methods and tools for the on-site power operations workers to prevent accidents caused by the operations personnel's safety consciousness, and improve the safety level of the substation site operations.

\section{ACKNOWLEDGEMENT}

This paper is sponsored by Science Foundation of State Grid Corporation of China (GN: XXB17201200315).

\section{REFERENCES}

[1] China Electricity Regulatory Bureau. Electricity Regulatory Annual Report (2008). Beijing: China Electricity Regulatory Bureau, 2009.

[2] China Electricity Regulatory Bureau. Electricity Regulatory Annual Report (2009).Beijing: China Electricity Regulatory Bureau, 2010.

[3] China Electricity Regulatory Bureau. Electricity Regulatory Annual Report (2010).Beijing: China Electricity Regulatory Bureau, 2011.

[4] State Grid Corporation Safety Supervision Department. Production Safety accident reports (2009).Beijing: State Grid Corporation, 2010.

[5] State Grid Corporation Safety Supervision Department. Production Safety accident reports (2010).Beijing: State Grid Corporation, 2011.

[6] Wei Zhiqiang, Ji Xiaopeng. \& Wang Peng. 2006. Real-time moving object detection for video monitoring systems. Journal of Systems Engineering and Electronics, 17(4): 731-736.

[7] Tian Tian, Zhou Bing. \& Li Bo. 2003. Optical Flow Computation Based on Analytic Wavelet. Journal of Beijing University of Aeronautics and Astronautics, 29 (6):548-551.

[8] Wei Jingzhi. 2008. Research on UWB wireless Networks. Beijing: Beijing university of Posts and Telecommunications.

[9] XIAO Zhu, WANG Yongchao, TIAN Bin. 2011. Development and Prospect of Ultra-Wideband Localization Research and Application. Acta Electronica Sinica, 39(1):133-141.

[10]Ding Rui, Qian Zhihong. \& Wang Xue. 2010. UWB Positioning System Based on Joint TOA and DOA Estimation. Journal of Electronics \& Information Technology,2: 313-317. 